\title{
Eight cubes of primes and powers of 2
}

\author{
by \\ Zhixin Liu and Guangshi Lü (Ji'nan)
}

1. Introduction. In 1951 and 1953, Linnik [16, 17 proved that each large even integer $N$ is a sum of two primes and a bounded number of powers of 2 ,

$$
N=p_{1}+p_{2}+2^{v_{1}}+\cdots+2^{v_{k}},
$$

where $p$ and $v$, with or without subscripts, denote a prime number and a positive integer respectively. Later Gallagher [3] established a stronger result by a different method. An explicit value for the number $k$ of powers of 2 was first established by Liu, Liu and Wang [21, who found that $k=54000$ is acceptable. This value was subsequently improved by $\mathrm{Li}$ [12, Wang [30] and $\mathrm{Li}$ [13. In 2002, Heath-Brown and Puchta [6] applied a rather different approach to this problem and showed that $k=13$ is acceptable. In 2003, Pintz and Ruzsa [25] announced that $k=8$ is acceptable.

In 1999, Liu, Liu and Zhan 22 proved that every large even integer $N$ can be written as a sum of four squares of primes and a bounded number of powers of 2 ,

$$
N=p_{1}^{2}+p_{2}^{2}+p_{3}^{2}+p_{4}^{2}+2^{v_{1}}+\cdots+2^{v_{k}} .
$$

Later Liu and Liu [18] showed that $k=8330$ is acceptable. This value was subsequently improved by Liu and Lü [23] and Li [14].

In 1938, Hua [7] proved that each large odd integer is the sum of nine cubes of primes. It seems reasonable to conjecture that every sufficiently large integer satisfying some necessary congruence conditions is the sum of eight cubes of primes, i.e.

$$
N=p_{1}^{3}+p_{2}^{3}+\cdots+p_{8}^{3},
$$

but unfortunately, such a conjecture is out of reach at present.

2010 Mathematics Subject Classification: Primary 11P32, 11P05, 11P55.

Key words and phrases: additive theory of prime numbers, circle method, exponential sums over primes. 
Motivated by this conjecture and the above works of Linnik and Gallagher for two primes and powers of 2, and the result of Liu, Liu and Zhan for four squares of primes and powers of 2, we extend the above results (1.1) and 1.2 to sums of eight cubes of primes and powers of 2, i.e.

$$
N=p_{1}^{3}+\cdots+p_{8}^{3}+2^{v_{1}}+\cdots+2^{v_{k}} .
$$

In 2000, Liu and Liu [20] proved that such a $k$ exists.

In this paper we bound the value of $k$ in $(1.4)$ by proving the following theorem.

THEOREM 1.1. Every large even integer is a sum of eight cubes of primes and 358 powers of 2 .

There are other approximations to the conjecture 1.3 , and our theorem can be compared with them. In [31, Wooley got an upper bound for the exceptional set for 1.3$)$ : he showed that with at most $O\left(N^{11 / 36+\varepsilon}\right)$ exceptions, all positive even integers not exceeding $N$ can be written as in 1.3$)$. Later Kumchev [10] improved this estimate to $O\left(N^{23 / 84+\varepsilon}\right)$. Roth [28] proved that every large integer $N$ can be written as

$$
N=m^{3}+p_{2}^{3}+\cdots+p_{8}^{3}
$$

with a positive integer $m$. Brüdern [1] combined the circle method with sieves to show that (1.5) is solvable when $m$ is a product $P_{4}$ of at most four primes. Kawada [9] improved the above $P_{4}$ to $P_{3}$.

Notation. As usual, $\varphi(n)$ and $\Lambda(n)$ denote the Euler totient function and the von Mangoldt function, respectively. We write $N$ for a large integer, and $L=\log N$. Further, $r \sim R$ means $R<r \leq 2 R$, and $A \asymp B$ means $c_{1} A \leq B \leq c_{2} A$. The letters $\varepsilon$ and $A$ denote positive constants, which are arbitrarily small and arbitrarily large, respectively.

2. Outline of the method. Here we outline the proof of Theorem 1.1 In order to apply the circle method, we set

$$
P=N^{1 / 9-2 \varepsilon}, \quad Q=N^{8 / 9+\varepsilon} .
$$

By Dirichlet's lemma ([29, Lemma 2.1]), each $\alpha \in[1 / Q, 1+1 / Q]$ may be written in the form

$$
\alpha=a / q+\lambda, \quad|\lambda| \leq 1 / q Q,
$$

for some integers $a, q$ with $1 \leq a \leq q \leq Q$ and $(a, q)=1$. Denote by $\mathcal{M}(a, q)$ the set of $\alpha$ satisfying 2.2 , and define the major arcs $\mathcal{M}$ and the minor 
$\operatorname{arcs} C(\mathcal{M})$ as follows:

$$
\mathcal{M}:=\bigcup_{1 \leq q \leq P} \bigcup_{\substack{1 \leq a \leq q \\(a, q)=1}} \mathcal{M}(a, q), \quad C(\mathcal{M})=\left[\frac{1}{Q}, 1+\frac{1}{Q}\right] \backslash \mathcal{M} .
$$

It follows from $2 P \leq Q$ that the major arcs $\mathcal{M}(a, q)$ are mutually disjoint.

As in [27], let $\delta=10^{-4}$, and

$$
U=\left(\frac{N}{16(1+\delta)}\right)^{1 / 3}, \quad V=U^{5 / 6} .
$$

As usual in the circle method, let

$$
\begin{gathered}
S(\alpha)=\sum_{p \sim U}(\log p) e\left(p^{3} \alpha\right), \quad T(\alpha)=\sum_{p \sim V}(\log p) e\left(p^{3} \alpha\right), \\
G(\alpha)=\sum_{2^{v} \leq N} e\left(2^{v} \alpha\right)=\sum_{v \leq \log _{2} N} e\left(2^{v} \alpha\right)
\end{gathered}
$$

and

$$
r_{k}(N)=\sum_{\substack{N=p_{1}^{3}+\cdots+p_{8}^{3}+2^{v_{1}}+\cdots+2^{v_{k}} \\ p_{1}, \ldots, p_{4} \sim U, p_{5}, \ldots, p_{8} \sim V}}\left(\log p_{1}\right) \ldots\left(\log p_{8}\right) .
$$

Then $r_{k}(N)$ can be written as

$$
\begin{aligned}
r_{k}(N) & =\int_{0}^{1} S^{4}(\alpha) T^{4}(\alpha) G^{k}(\alpha) e(-N \alpha) d \alpha \\
& =\left\{\int_{\mathcal{M}}+\int_{C(\mathcal{M})}\right\} S^{4}(\alpha) T^{4}(\alpha) G^{k}(\alpha) e(-N \alpha) d \alpha .
\end{aligned}
$$

To handle the integral on the major arcs, we prove the following lemma.

Lemma 2.1. Let $\mathcal{M}$ be as in (2.3), with $P$ and $Q$ determined by (2.1). Then for $N / 2 \leq n \leq N$, we have

$$
\int_{\mathcal{M}} S^{4}(\alpha) T^{4}(\alpha) e(-n \alpha) d \alpha=\frac{1}{3^{8}} \mathfrak{S}(n) J(n)+O\left(U V^{4} L^{-1}\right) .
$$

Here $\mathfrak{S}(n)$ is a singular series, which is defined by

$$
\mathfrak{S}(n):=\sum_{q=1}^{\infty} \frac{1}{\varphi^{8}(q)} \sum_{\substack{a=1 \\(a, q)=1}}^{q}\left(\sum_{\substack{h=1 \\(h, q)=1}}^{q} e\left(\frac{a h^{3}}{q}\right)\right)^{8} e\left(-\frac{a n}{q}\right),
$$

and satisfies $\mathfrak{S}(n) \gg 1$ for $n \equiv 0(\bmod 2) . J(n)$ is defined as

$$
J(n):=\sum_{\substack{m_{1}+\ldots+m_{8}=n \\ U^{3}<m_{1}, \ldots, m_{4} \leq 8 U^{3}, V^{3}<m_{5}, \ldots, m_{8} \leq 8 V^{3}}}\left(m_{1} \ldots m_{8}\right)^{-2 / 3},
$$


and satisfies

$$
U V^{4} \ll J(n) \ll U V^{4}
$$

In this paper, the constants in the $\gg$ and $\ll$ symbols are of importance. If we write $\mathfrak{S}(n)>C_{1}$ and $J(n)>C_{2} U V^{4}$, in the following parts, we determine explicit values of $C_{1}, C_{2}$.

A crucial step in bounding the contributions of minor arcs is an upper bound for the number of solutions of the equation

$$
n=p_{1}^{3}+\cdots+p_{4}^{3}-p_{5}^{3}-\cdots-p_{8}^{3}, \quad 0 \leq|n| \leq N .
$$

We quote the following lemma.

LEMMA 2.2. Let $n \equiv 0(\bmod 2)$ be an integer, and $\rho(n)$ the number of representations of $n$ in the form (2.13) subject to

$$
p_{1}, p_{2}, p_{5}, p_{6} \sim U, \quad p_{3}, p_{4}, p_{7}, p_{8} \sim V .
$$

Then for all $0 \leq|n| \leq N$,

$$
\rho(n) \leq b U V^{4} L^{-8}
$$

with $b=268096$.

The inequality (2.15) is (2.6) in Ren [26], obtained by sieve methods, and the value of $b$ is determined in Ren [27].

On the minor arcs, we also need estimates for the measure of the set

$$
\mathcal{E}_{\lambda}=\left\{\alpha \in(0,1]:|G(\alpha)| \geq \lambda \log _{2} N\right\} .
$$

The following lemma is due to Heath-Brown and Puchta [6].

LEMMA 2.3. Let

$$
G_{h}(\alpha)=\sum_{0 \leq n \leq h-1} e\left(\alpha 2^{n}\right), \quad F(\xi, h)=\frac{1}{2^{h}} \sum_{r=0}^{2^{h}-1} \exp \left[\xi \operatorname{Re}\left(G_{h}\left(\frac{r}{2^{h}}\right)\right)\right] .
$$

Then

$$
\operatorname{meas}\left(\mathcal{E}_{\lambda}\right) \leq N^{-E(\lambda)}
$$

where

$$
E(\lambda)=\frac{\xi \lambda}{\log 2}-\frac{\log F(\xi, h)}{h \log 2}-\frac{\varepsilon}{\log 2}
$$

for any $h \in \mathbb{N}, \xi>0$ and $\varepsilon>0$.

On the minor arcs, the results of Kumchev [10] on exponential sums over primes will also be applied. The following lemma is Theorem 3 of [10] for $k=3$. 
Lemma 2.4 (Kumchev). Let $\alpha=a / q+\lambda$ subject to $1 \leq a \leq q$, $(a, q)=1$, and $|\lambda| \leq 1 / q Q$, with $Q=U^{12 / 7}$, and let $S(\alpha)$ be defined in 2.5). Then

$$
S(\alpha) \ll U^{1-\varrho+\varepsilon}+\frac{q^{\varepsilon} U L^{c}}{\sqrt{q\left(1+|\lambda| U^{3}\right)}}
$$

with $\varrho=1 / 14$.

We deduce Theorem 1.1 from some lemmas in Section 3. In Section 4, we give the proof of Lemma 2.1. In Sections 5 and 6, we give the value of $C_{1}$ and the proofs of three lemmas, respectively.

3. The proof of Theorem 1.1. We need the following five lemmas.

LEMMA 3.1. Let

$$
\Xi(N, k)=\left\{(1-\delta) N \leq n \leq N: n=N-2^{\nu_{1}}-\cdots-2^{\nu_{k}}\right\},
$$

with $k \geq 2$. Then for $N \equiv 0(\bmod 2)$,

$$
\sum_{\substack{n \in \Xi(N, k) \\ n \equiv 0(\bmod 2)}} 1 \geq(1-\varepsilon)\left(\log _{2} N\right)^{k} .
$$

Proof. The proof is straightforward, so we omit the details.

Lemma 3.2. For $n \equiv 0(\bmod 2)$, we have $\mathfrak{S}(n)>C_{1}$ with

$$
C_{1}=0.00557795824
$$

while for $n \not \equiv 0(\bmod 2)$, we have $\mathfrak{S}(n)=0$.

Proof. We will prove this in Section 5.

Lemma 3.3. For $(1-\delta) N \leq n \leq N$, we have $J(n)>C_{2} U V^{4}$, with

$$
C_{2}=78.15467793 \text {. }
$$

Proof. We will determine the value of $C_{2}$ in Section 4 .

Lemma 3.4. Let $C(\mathcal{M})$ be as in $(2.3)$, with $P$ and $Q$ determined by (2.1), and $S(\alpha)$ be as in 2.5$)$. Then

$$
\max _{\alpha \in C(\mathcal{M})}|S(\alpha)| \ll N^{1 / 3-1 / 42+\varepsilon} .
$$

Proof. By Dirichlet's lemma on rational approximations, each real number $\alpha \in C(\mathcal{M})$ can be written as $\alpha=a / q+\lambda$ with $(a, q)=1$ and

$$
1 \leq q \leq Q_{0}=N^{4 / 7}, \quad|\lambda| \leq 1 / q Q_{0} .
$$


If $q \leq P=N^{1 / 9-2 \varepsilon}$, since $\alpha \in C(\mathcal{M})$, we have $|\lambda|>1 / q Q$; otherwise $q>P$. In either case,

$$
\sqrt{q\left(1+|\lambda| U^{3}\right)}>\min \left(P^{1 / 2},\left(U^{3} / Q\right)^{1 / 2}\right)=N^{1 / 18-\varepsilon} .
$$

By Lemma 2.4, the conclusion follows.

In order to apply Lemma 2.3 , we need to find an optimal $\lambda$ such that $E(\lambda)>19 / 21$. Thus we have to compute

$$
F(\xi, h)=\frac{1}{2^{h}} \sum_{r=0}^{2^{h}-1} \exp \left[\xi \operatorname{Re}\left(G_{h}\left(\frac{r}{2^{h}}\right)\right)\right],
$$

and optimize for $\xi$ and $h$. We can take $\xi=1.59, h=23$ in Lemma 2.3 to get

Lemma 3.5. Let $E(\lambda)$ be as in Lemma 2.3, Then

$$
E(0.965411)>\frac{19}{21}+10^{-10} .
$$

Proof of Theorem 1.1. Let $N \equiv 0(\bmod 2)$, let $\mathcal{E}_{\lambda}$ be as in $(2.16)$ and $\mathcal{M}$ as in (2.3), with $P$ and $Q$ determined by (2.1). Then, by (2.8),

$$
\begin{aligned}
r_{k}(N) & =\int_{0}^{1} S^{4}(\alpha) T^{4}(\alpha) G^{k}(\alpha) e(-N \alpha) d \alpha \\
& =\int_{\mathcal{M}}+\int_{C(\mathcal{M}) \cap \mathcal{E}_{\lambda}}+\int_{C(\mathcal{M}) \cap C\left(\mathcal{E}_{\lambda}\right)} .
\end{aligned}
$$

Introducing the notation $\Xi(N, k)$ and then applying Lemma 2.1, we see that the first integral on the right-hand side of $(3.6)$ is

$$
\begin{aligned}
\sum_{n \in \Xi(N, k)} \int_{\mathcal{M}} S^{4}(\alpha) T^{4}(\alpha) & e(-n \alpha) d \alpha \\
& =\frac{1}{3^{8}} \sum_{n \in \Xi(N, k)} \mathfrak{S}(n) J(n)+O\left(U V^{4} L^{k-1}\right) \\
& \geq \frac{1}{3^{8}} C_{1} C_{2} U V^{4} \sum_{n \in \Xi(N, k)} 1+O\left(U V^{4} L^{k-1}\right) \\
& \geq \frac{1}{3^{8}} C_{1} C_{2}(1-\varepsilon) U V^{4}\left(\log _{2} N\right)^{k}
\end{aligned}
$$

where in the last two inequalities we have used Lemmas 3.13 .3 .

With Lemma 3.4, the second integral satisfies

$$
\int_{C(\mathcal{M}) \cap \mathcal{E}_{\lambda}} \ll N^{-E(\lambda)}\left(N^{1 / 3-1 / 42+\varepsilon}\right)^{4} V^{4}\left(\log _{2} N\right)^{k} \ll U V^{4} L^{k-1} .
$$


By using the definition of $\mathcal{E}_{\lambda}$ and Lemma 2.2, the last integral in 3.6 can be estimated as follows:

$$
\begin{aligned}
\int_{C(\mathcal{M}) \cap C\left(\mathcal{E}_{\lambda}\right)} & \leq\left(\lambda \log _{2} N\right)^{k} \int_{0}^{1}|S(\alpha) T(\alpha)|^{4} d \alpha \\
& \leq\left(\lambda \log _{2} N\right)^{k}(\log (2 U))^{4}(\log (2 V))^{4} \rho(0) \\
& \leq\left(\lambda \log _{2} N\right)^{k}\left(\frac{1}{3}\right)^{4}\left(\frac{5}{18}\right)^{4} b U V^{4},
\end{aligned}
$$

where in the last inequality we have used Lemma 2.2 and the definition of $\rho(n)$.

Inserting (3.7)-(3.9) into (3.6), we get

$$
\begin{aligned}
r_{k}(N) \geq & \left(\left(\frac{1}{3}\right)^{8} C_{1} C_{2}-\left(\frac{1}{3}\right)^{4}\left(\frac{5}{18}\right)^{4} b \lambda^{k}\right)(1-\varepsilon) U V^{4}\left(\log _{2} N\right)^{k} \\
& +O\left(U V^{4} L^{k-1}\right) .
\end{aligned}
$$

When $k \geq 358$ and $\varepsilon=10^{-10}$, we obtain

$$
r_{k}(N)>1.3 \cdot 10^{-7} U V^{4}\left(\log _{2} N\right)^{k} .
$$

Recalling the definition of $U$ and $V$, we conclude that every sufficiently large even integer $N$ can be expressed in the form (1.4). This completes the proof of Theorem 1.1 .

4. The major arcs: proof of Lemma 2.1. For $\chi$ a character modulo $q$, define

$$
C(\chi, a):=\sum_{h=1}^{q} \bar{\chi}(h) e\left(\frac{a h^{3}}{q}\right), \quad C(q, a):=C\left(\chi^{0}, a\right) .
$$

If $\chi_{1}, \ldots, \chi_{8}$ are characters modulo $q$, then we write

$$
\begin{aligned}
B\left(n, q ; \chi_{1}, \ldots, \chi_{8}\right) & :=\sum_{\substack{a=1 \\
(a, q)=1}}^{q} e\left(-\frac{a n}{q}\right) C\left(\chi_{1}, a\right) \ldots C\left(\chi_{8}, a\right), \\
B(n, q) & :=B\left(n, q ; \chi^{0}, \ldots, \chi^{0}\right) .
\end{aligned}
$$

The following lemma is important in proving Lemma 2.1 .

Lemma 4.1. Let $\chi_{i}$ with $i=1, \ldots, 8$ be primitive characters modulo $r_{i}$, $r_{0}=\left[r_{1}, \ldots, r_{8}\right]$, and $\chi^{0}$ be the principal character modulo $q$. Then

$$
\sum_{q \leq z, r_{0} \mid q} \frac{1}{\varphi^{8}(q)}\left|B\left(n, q ; \chi_{1} \chi^{0}, \ldots, \chi_{8} \chi^{0}\right)\right| \ll r_{0}^{-3+\varepsilon} \log ^{c} z .
$$

Proof. It is similar to that of Lemma 7 in [11, so we omit the details. 
To state other preliminaries, we need to introduce some extra notations. For $i=1,2$ and $W$ equal to $U$ or $V$ respectively, we define

$$
\begin{aligned}
V_{i}(\lambda) & :=\sum_{m \sim W} e\left(m^{3} \lambda\right), \\
W_{i}(\chi, \lambda) & :=\sum_{p \sim W}(\log p) \chi(p) e\left(p^{3} \lambda\right)-\delta_{\chi} \sum_{m \sim W} e\left(m^{3} \lambda\right),
\end{aligned}
$$

where $\delta_{\chi}=1$ or 0 according as $\chi$ is principal or not. Define

$$
\begin{aligned}
J_{i}(g) & :=\sum_{r \leq P}[g, r]^{-3+\varepsilon} \sum_{\chi \bmod r}^{*} \max _{|\lambda| \leq 1 / r Q}\left|W_{i}(\chi, \lambda)\right|, \\
K(g) & :=\sum_{r \leq P}[g, r]^{-3+\varepsilon} \sum_{\chi \bmod r}^{*}\left(\int_{-1 / r Q}^{1 / r Q}\left|W_{1}(\chi, \lambda)\right|^{2} d \lambda\right)^{1 / 2} .
\end{aligned}
$$

Estimates for $J_{i}(i=1,2)$ and $K$ are needed in later arguments. In particular, the following three lemmas will be important to deal with enlarged major arcs.

Lemma 4.2. Let $U, V$ be as in 2.4), and let $P, Q$ satisfy (2.1). Then

$$
J_{i}(g) \ll g^{-3+\varepsilon} W L^{c} .
$$

Lemma 4.3. Let $U, P, Q$ be as in Lemma 4.2. If $g=1$, then (4.8) can be improved to

$$
J_{1}(1) \ll U L^{-A}
$$

where $A>0$ is arbitrary.

Lemma 4.4. Let $U, P, Q$ be as in Lemma 4.2. Then

$$
K(g) \ll g^{-3+\varepsilon} U^{-1 / 2} L^{c} .
$$

We will prove Lemmas 4.24 .4 in Section 6 .

Proof of Lemma 2.1. Introducing Dirichlet characters, we can rewrite the exponential sums $S(\alpha)$ and $T(\alpha)$ as

$$
\begin{aligned}
& S\left(\frac{a}{q}+\lambda\right)=\frac{C(q, a)}{\varphi(q)} V_{1}(\lambda)+\frac{1}{\varphi(q)} \sum_{\chi \bmod q} C(\chi, a) W_{1}(\chi, \lambda), \\
& T\left(\frac{a}{q}+\lambda\right)=\frac{C(q, a)}{\varphi(q)} V_{2}(\lambda)+\frac{1}{\varphi(q)} \sum_{\chi \bmod q} C(\chi, a) W_{2}(\chi, \lambda) .
\end{aligned}
$$

Thus

$$
\int_{\mathcal{M}} S^{4}(\alpha) T^{4}(\alpha) e(-n \alpha) d \alpha=\sum_{0 \leq i \leq 4} \sum_{0 \leq j \leq 4} C_{4}^{i} C_{4}^{j} I_{i j}
$$


where

$$
\begin{aligned}
I_{i j}= & \sum_{q \leq P} \frac{1}{\varphi^{8}(q)} \sum_{\substack{a=1 \\
(a, q)=1}}^{q} C^{8-i-j}(q, a) e\left(-\frac{a n}{q}\right) \int_{-1 / q Q}^{1 / q Q} V_{1}^{4-i}(\lambda) V_{2}^{4-j}(\lambda) \\
& \times\left\{\sum_{\chi \bmod q} C(\chi, a) W_{1}(\chi, \lambda)\right\}^{i}\left\{\sum_{\chi \bmod q} C(\chi, a) W_{2}(\chi, \lambda)\right\}^{j} e(-n \lambda) d \lambda .
\end{aligned}
$$

We will prove that $I_{00}$ gives the main term, and the others the error term.

We begin with $I_{00}$, which we expect to be the main term:

$$
\begin{aligned}
I_{00}= & \sum_{q \leq P} \frac{1}{\varphi^{8}(q)} \sum_{\substack{a=1 \\
(a, q)=1}}^{q} C^{8}(q, a) e\left(-\frac{a n}{q}\right) \\
& \times \int_{-1 / q Q}^{1 / q Q} V_{1}^{4}(\lambda) V_{2}^{4}(\lambda) e(-n \lambda) d \lambda .
\end{aligned}
$$

By Lemma 7.11 of $[8]$,

$$
V_{i}(\lambda)=\int_{W}^{2 W} e\left(\lambda u^{3}\right) d u+O(1)=\frac{1}{3} \sum_{W^{3}<m \leq 8 W^{3}} m^{-2 / 3} e(m \lambda)+O(1) .
$$

Using this and the elementary estimate

$$
\sum_{W^{3}<m \leq 8 W^{3}} m^{-2 / 3} e(m \lambda) \ll \min \left(W, W^{-2}|\lambda|^{-1}\right),
$$

we have

$$
\begin{aligned}
I_{00}=\frac{1}{3^{8}} \sum_{q \leq P} \frac{B(n, q)}{\varphi^{8}(q)} \int_{-1 / q Q}^{1 / q Q}\left(\sum_{U^{3}<m \leq 8 U^{3}} m^{-2 / 3} e(m \lambda)\right)^{4} \\
\times\left(\sum_{V^{3}<m \leq 8 V^{3}} m^{-2 / 3} e(m \lambda)\right)^{4} e(-n \lambda) d \lambda \\
+O\left(\sum_{q \leq P} \frac{|B(n, q)|}{\varphi^{8}(q)} \int_{-1 / q Q}^{1 / q Q}\left|\sum_{U^{3}<m \leq 8 U^{3}} m^{-2 / 3} e(m \lambda)\right|^{4}\right. \\
\left.\quad \times\left|\sum_{V^{3}<m \leq 8 V^{3}} m^{-2 / 3} e(m \lambda)\right|^{3} d \lambda\right) .
\end{aligned}
$$

By 4.16) and Lemma 4.1 with $r_{0}=1$, the $O$-term in 4.17) can be estima- 
ted as

$$
\begin{aligned}
& \ll \sum_{q \leq P} \frac{|B(n, q)|}{\varphi^{8}(q)}\left(\int_{0}^{U^{-3}} U^{4} V^{3} d \lambda+\int_{U^{-3}}^{V^{-3}} U^{-8} \lambda^{-4} V^{3} d \lambda\right. \\
&\left.+\int_{V^{-3}}^{\infty} U^{-8} \lambda^{-4} V^{-6} \lambda^{-3} d \lambda\right) \\
& \ll L^{c}\left(U V^{3}+U V^{3}+U^{-8} V^{12}\right) \ll U V^{3} L^{c} \ll U V^{4} L^{-1} .
\end{aligned}
$$

Now we extend the integral in the main term of 4.17 to $[-1 / 2,1 / 2]$; by a similar argument we see that the resulting error can be estimated as

$$
\ll L^{c} \int_{1 / P Q}^{1 / 2}\left(U^{-2} \lambda^{-1}\right)^{4} V^{4} d \lambda \ll L^{c} U^{-8} V^{4}(P Q)^{3} \ll U V^{4} L^{-1},
$$

which is acceptable by the choice of $P$ and $Q$. Thus the main term of 4.17 becomes

$$
\begin{array}{r}
\frac{1}{3^{8}} \sum_{q \leq P} \frac{B(n, q)}{\varphi^{8}(q)} \sum_{\substack{m_{1}+\cdots+m_{8}=n \\
U^{3}<m_{1}, \ldots, m_{4} \leq 8 U^{3}, V^{3}<m_{5}, \ldots, m_{8} \leq 8 V^{3}}}\left(m_{1} \ldots m_{8}\right)^{-2 / 3}+O\left(U V^{4} L^{-1}\right) \\
=\frac{1}{3^{8}} \sum_{q \leq P} \frac{B(n, q)}{\varphi^{8}(q)} J(n)+O\left(U V^{4} L^{-1}\right),
\end{array}
$$

where $J(n)$ is defined by 2.11 .

The first sum above is $\mathfrak{S}(n)+O\left(L^{-1}\right)$. The domain of the second sum, $J(n)$, can be written as

$$
\mathfrak{D}=\left\{\left(m_{1}, \ldots, m_{8}\right): U^{3}<m_{1}, \ldots, m_{4} \leq 8 U^{3}, V^{3}<m_{5}, \ldots, m_{8} \leq 8 V^{3}\right\},
$$

with $m_{1}=n-m_{2}-\cdots-m_{8}$.

To bound this sum from below, if we define

$$
\mathfrak{D}^{*}=\left\{\left(m_{2}, \ldots, m_{8}\right): \frac{8}{3} U^{3}<m_{2}, \ldots, m_{4} \leq 5 U^{3}, V^{3}<m_{5}, \ldots, m_{8} \leq 8 V^{3}\right\},
$$

we can deduce from $(1-\delta) N<n \leq N$ and 2.4 that

$$
U^{3}<m_{1}=n-m_{2}-\cdots-m_{8} \leq 8 U^{3} .
$$

Thus $\mathfrak{D}^{*}$ is a subset of $\mathfrak{D}$, and consequently

$$
\begin{aligned}
& J(n) \geq \sum_{\substack{U^{3}<m_{1} \leq 8 U^{3}, \frac{8}{3} U^{3}<m_{2}, m_{3} \leq 5 U^{3} \\
V^{3}<m_{5}, \ldots, m_{8} \leq 8 V^{3}}}\left(m_{1} \ldots m_{8}\right)^{-2 / 3} \\
& \geq\left(5 U^{3}\right)^{-2 / 3}\left\{5^{1 / 3}-\left(\frac{8}{3}\right)^{1 / 3}\right\}^{2} 3^{7} U^{3} V^{4} \geq 78.15467793 U V^{4} .
\end{aligned}
$$

So, we get $C_{2}=78.15467793$. 
It remains to estimate $I_{i j}(0 \leq i, j \leq 4$, not both zero). We shall first treat $I_{44}$, the most complicated one, and the others are similar:

$$
\begin{aligned}
I_{44}= & \sum_{q \leq P} \frac{1}{\varphi^{8}(q)} \sum_{\substack{a=1 \\
(a, q)=1}}^{q} e\left(-\frac{a n}{q}\right) \int_{-1 / q Q}^{1 / q Q}\left\{\sum_{\chi \bmod q} C(\chi, a) W_{1}(\chi, \lambda)\right\}^{4} \\
& \times\left\{\sum_{\chi \bmod q} C(\chi, a) W_{2}(\chi, \lambda)\right\}^{4} e(-n \lambda) d \lambda \\
= & \sum_{q \leq P} \frac{1}{\varphi^{8}(q)} \sum_{\chi_{1} \bmod q} \ldots \sum_{\chi_{8} \bmod q} \sum_{\substack{a=1 \\
(a, q)=1}}^{q} C\left(\chi_{1}, a\right) \ldots C\left(\chi_{8}, a\right) e\left(-\frac{a n}{q}\right) \\
& \times \int_{-1 / q Q}^{1 / q Q} W_{1}\left(\chi_{1}, \lambda\right) \ldots W_{1}\left(\chi_{4}, \lambda\right) W_{2}\left(\chi_{5}, \lambda\right) \ldots W_{2}\left(\chi_{8}, \lambda\right) e(-n \lambda) d \lambda \\
= & \sum_{r_{1} \leq P} \ldots \sum_{r_{8} \leq P} \sum_{\chi_{1} \bmod r_{1}}^{*} \ldots \sum_{\chi_{8} \bmod r_{8}}^{*} \sum_{r_{0} \mid P} \frac{B\left(n, q ; \chi_{1} \chi^{0}, \ldots, \chi_{8} \chi^{0}\right)}{\varphi^{8}(q)} \\
& \times \int_{-1 / q Q}^{1 / q Q} W_{1}\left(\chi_{1}, \lambda\right) \ldots W_{1}\left(\chi_{4}, \lambda\right) W_{2}\left(\chi_{5}, \lambda\right) \ldots W_{2}\left(\chi_{8}, \lambda\right) e(-n \lambda) d \lambda,
\end{aligned}
$$

where $\chi_{0}$ is the principal character modulo $q, r_{0}=\left[r_{1}, \ldots, r_{8}\right]$, and the sum $\sum^{*}$ is taken over all primitive characters. Suppose that $\chi_{k}^{*}$ is the primitive character modulo $r_{k}$ with $r_{k} \mid q$, inducing $\chi_{k}$. Thus we may write $\chi_{k}=\chi_{k}^{*} \chi^{0}$. It is easy to see that $W\left(\chi_{k}, \lambda\right)=W\left(\chi_{k}^{*}, \lambda\right)$. By Lemma 4.1 and Cauchy's inequality, we have

$$
\begin{aligned}
& \left|I_{44}\right| \ll L^{c} \sum_{r_{1} \leq P} \sum_{\chi_{1} \bmod r_{1}}^{*} \max _{|\lambda| \leq 1 / r_{1} Q}\left|W_{1}\left(\chi_{1}, \lambda\right)\right| \sum_{r_{2} \leq P} \sum_{\chi_{2}}^{*}{\operatorname{mad} r_{2}}_{|\lambda| \leq 1 / r_{2} Q}\left|W_{1}\left(\chi_{2}, \lambda\right)\right| \\
& \times \sum_{r_{3} \leq P} \sum_{\chi_{3} \bmod r_{3}}^{*}\left(\int_{-1 / r_{3} Q}^{1 / r_{3} Q}\left|W_{1}\left(\chi_{3}, \lambda\right)\right|^{2} d \lambda\right)^{1 / 2} \\
& \times \sum_{r_{4} \leq P} \sum_{\chi_{4} \bmod r_{4}}^{*}\left(\int_{-1 / r_{4} Q}^{1 / r_{4} Q}\left|W_{1}\left(\chi_{4}, \lambda\right)\right|^{2} d \lambda\right)^{1 / 2} \\
& \times \sum_{r_{5} \leq P} \sum_{\chi_{5} \bmod r_{5}}^{*} \max _{|\lambda| \leq 1 / r_{5} Q}\left|W_{2}\left(\chi_{5}, \lambda\right)\right| \sum_{r_{6} \leq P} \sum_{\chi_{6} \bmod r_{6}}^{*} \max _{|\lambda| \leq 1 / r_{6} Q}\left|W_{2}\left(\chi_{6}, \lambda\right)\right| \\
& \times \sum_{r_{7} \leq P \chi_{7} \bmod r_{7}} \sum_{|\lambda| \leq 1 / r_{7} Q}^{*}\left|W_{2}\left(\chi_{7}, \lambda\right)\right| \sum_{r_{8} \leq P} r_{0}^{-3+\varepsilon} \sum_{\chi_{8} \bmod r_{8}}^{*} \max _{|\lambda| \leq 1 / r_{8} Q}\left|W_{2}\left(\chi_{8}, \lambda\right)\right| .
\end{aligned}
$$


Now we introduce an iterative procedure to bound the above sums over $r_{8}, \ldots, r_{1}$ consecutively. Since $r_{0}=\left[r_{1}, \ldots, r_{8}\right]=\left[\left[r_{1}, \ldots, r_{7}\right], r_{8}\right]$, we use Lemma 4.2 four times, Lemma 4.4 twice, Lemma 4.2 once, and Lemma 4.3 once to get

$$
\begin{aligned}
\left|I_{44}\right| \ll & L^{c} \sum_{r_{1} \leq P} \sum_{\chi_{1} \bmod r_{1}}^{*} \max _{|\lambda| \leq 1 / r_{1} Q}\left|W_{1}\left(\chi_{1}, \lambda\right)\right| \sum_{r_{2} \leq P} \sum_{\chi_{2} \bmod r_{2}}^{*} \max _{|\lambda| \leq 1 / r_{2} Q}\left|W_{1}\left(\chi_{2}, \lambda\right)\right| \\
& \times \sum_{r_{3} \leq P} \sum_{\chi_{3} \bmod r_{3}}^{*}\left(\int_{-1 / r_{3} Q}^{1 / r_{3} Q}\left|W_{1}\left(\chi_{3}, \lambda\right)\right|^{2} d \lambda\right)^{1 / 2} \\
& \times \sum_{r_{4} \leq P} \sum_{\chi_{4} \bmod r_{4}}^{*}\left(\int_{-1 / r_{4} Q}^{1 / r_{4} Q}\left|W_{1}\left(\chi_{4}, \lambda\right)\right|^{2} d \lambda\right)^{1 / 2} \\
& \times\left[r_{1}, r_{2}, r_{3}, r_{4}\right]^{-3+\varepsilon} V^{4} L^{4 c} \\
\ll & L^{c} \sum_{r_{1} \leq P} \sum_{\chi_{1} \bmod r_{1}}^{*} \max _{|\lambda| \leq 1 / r_{1} Q}\left|W_{1}\left(\chi_{1}, \lambda\right)\right| \sum_{r_{2} \leq P} \sum_{\chi_{2} \bmod r_{2}}^{*} \max _{|\lambda| \leq 1 / r_{2} Q}\left|W_{2}\left(\chi_{2}, \lambda\right)\right| \\
& \times\left[r_{1}, r_{2}\right]^{-3+\varepsilon} U^{-1} V^{4} L^{6 c} \\
\ll & L^{c} \sum_{r_{1} \leq P} \sum_{\chi_{1} \bmod r_{1}}^{*} \max _{|\lambda| \leq 1 / r_{1} Q}\left|W_{1}\left(\chi_{1}, \lambda\right)\right| r_{1}^{-3+\varepsilon} V^{4} L^{7 c} \\
\ll & U V^{4} L^{-A+8 c} \ll U V^{4} L^{-1}
\end{aligned}
$$

for large $A>0$.

To get upper bounds for other terms, we need to estimate $V_{1}(\lambda)$ and $V_{2}(\lambda)$.

One easily gets

$$
\max _{|\lambda| \leq 1 / Q}\left|V_{i}(\lambda)\right| \ll W .
$$

By 4.15 and 4.16,

$$
\begin{aligned}
\int_{-1 / Q}^{1 / Q}\left|V_{i}(\lambda)\right|^{2} d \lambda \ll \int_{-1 / Q}^{1 / Q}\left(\left(\min \left(W^{-1}, W^{-2}|\lambda|^{-1}\right)\right)^{2}+O(1)\right) d \lambda \\
\ll \int_{0}^{W^{-3}} W^{2} d \lambda+\int_{W^{-3}}^{\infty}\left(W^{-2}|\lambda|^{-1}\right)^{2} d \lambda+\int_{-1 / Q}^{1 / Q} d \lambda \ll W^{-1},
\end{aligned}
$$

by the choices of $P$ and $Q$ in (2.1), and $W=U$ or $V$ as $i=1,2$. 
For all $I_{i j}, 0 \leq i, j \leq 4$, except $I_{00}$ and $I_{44}$,

$$
\begin{aligned}
I_{4 j}= & \sum_{q \leq P} \frac{1}{\varphi^{8}(q)} \sum_{\substack{a=1 \\
(a, q)=1}}^{q} C^{4-j}(q, a) e\left(-\frac{a n}{q}\right) \int_{-1 / q Q}^{1 / q Q} V_{2}^{4-j}(\lambda) \\
& \times\left\{\sum_{\chi \bmod q} C(\chi, a) W_{1}(\chi, \lambda)\right\}^{4}\left\{\sum_{\chi \bmod q} C(\chi, a) W_{2}(\chi, \lambda)\right\}^{j} e(-n \lambda) d \lambda,
\end{aligned}
$$

and

$$
\begin{aligned}
\left|I_{4 j}\right| \ll & L^{c} \sum_{r_{1} \leq P} \sum_{\chi_{1} \bmod r_{1}}^{*} \max _{|\lambda| \leq 1 / r_{1} Q}\left|W_{1}\left(\chi_{1}, \lambda\right)\right| \sum_{r_{2} \leq P} \sum_{\chi_{2} \bmod r_{2}}^{*} \max _{|\lambda| \leq 1 / r_{2} Q}\left|W_{1}\left(\chi_{2}, \lambda\right)\right| \\
& \times \sum_{r_{3} \leq P} \sum_{\chi_{3} \bmod r_{3}}^{*}\left(\int_{-1 / r_{3} Q}^{1 / r_{3} Q}\left|W_{1}\left(\chi_{3}, \lambda\right)\right|^{2} d \lambda\right)^{1 / 2} \\
& \times \sum_{r_{4} \leq P} \sum_{\chi_{4} \bmod r_{4}}^{*}\left(\int_{-1 / r_{4} Q}^{1 / r_{4} Q}\left|W_{1}\left(\chi_{4}, \lambda\right)\right|^{2} d \lambda\right)^{1 / 2} \\
& \times \sum_{r_{5} \leq P} \sum_{\chi_{5} \bmod r_{5}}^{*} \max _{|\lambda| \leq 1 / r_{5} Q}\left|W_{2}\left(\chi_{5}, \lambda\right)\right| \ldots \\
& \ldots \sum_{r_{4+j} \leq P} \sum_{\chi_{4+j} \bmod r_{4+j}}^{*} \max _{|\lambda| \leq 1 / r_{4+j} Q} r_{0}^{-3+\varepsilon}\left|W_{2}\left(\chi_{4+j}, \lambda\right)\right|\left(\max _{|\lambda| \leq 1 / Q}\left|V_{2}(\lambda)\right|\right)^{4-j} .
\end{aligned}
$$

Now we use (4.21) $4-j$ times, Lemma $4.2 j$ times, Lemma 4.4 twice, Lemma 4.2 once again, and Lemma 4.3 once to get

$$
\left|I_{4 j}\right| \ll U V^{4} L^{-A+(4+j) c} \ll U V^{4} L^{-1}
$$

for large $A>0$. We treat $\left|I_{3 j}\right|,\left|I_{2 j}\right|,\left|I_{1 j}\right|$ and $\left|I_{0 j}\right|$ by similar arguments:

$$
\begin{aligned}
\left|I_{3 j}\right| \ll & L^{c} \sum_{r_{1} \leq P} \sum_{\chi_{1} \bmod r_{1}}^{*} \max _{|\lambda| \leq 1 / r_{1} Q}\left|W_{1}\left(\chi_{1}, \lambda\right)\right| \sum_{r_{2} \leq P} \sum_{\chi_{2} \bmod r_{2}}^{*} \max _{|\lambda| \leq 1 / r_{2} Q}\left|W_{1}\left(\chi_{2}, \lambda\right)\right| \\
& \times \sum_{r_{3} \leq P} \sum_{\chi_{3} \bmod r_{3}}^{*}\left(\int_{-1 / r_{3} Q}^{1 / r_{3} Q}\left|W_{1}\left(\chi_{3}, \lambda\right)\right|^{2} d \lambda\right)^{1 / 2}\left(\int_{-1 / Q}^{1 / Q}\left|V_{1}(\lambda)\right|^{2} d \lambda\right)^{1 / 2} \\
& \times \sum_{r_{5} \leq P} \sum_{\chi_{5} \bmod r_{5}}^{*} \max _{|\lambda| \leq 1 / r_{5} Q}\left|W_{2}\left(\chi_{5}, \lambda\right)\right| \ldots \\
& \ldots \sum_{r_{4+j} \leq P} \sum_{\chi_{4+j} \bmod r_{4+j}}^{*} \max _{|\lambda| \leq 1 / r_{4+j} Q} r_{0}^{-3+\varepsilon}\left|W_{2}\left(\chi_{4+j}, \lambda\right)\right|\left(\max _{|\lambda| \leq 1 / Q}\left|V_{2}(\lambda)\right|\right)^{4-j} \\
& \ll U V^{4} L^{-1},
\end{aligned}
$$




$$
\begin{aligned}
&\left|I_{2 j}\right| \ll L^{c} \sum_{r_{1} \leq P} \sum_{\chi_{1} \bmod r_{1}}^{*} \max _{|\lambda| \leq 1 / r_{1} Q}\left|W_{1}\left(\chi_{1}, \lambda\right)\right| \\
& \times \sum_{r_{2} \leq P} \sum_{\chi_{2} \bmod r_{2}}^{*} \max _{|\lambda| \leq 1 / r_{2} Q}\left|W_{1}\left(\chi_{2}, \lambda\right)\right|\left(\int_{-1 / Q}^{1 / Q}\left|V_{1}(\lambda)\right|^{2} d \lambda\right) \\
& \times \sum_{r_{5} \leq P} \sum_{\chi_{5} \bmod r_{5}}^{*} \max _{|\lambda| \leq 1 / r_{5} Q}\left|W_{2}\left(\chi_{5}, \lambda\right)\right| \ldots \\
& \ldots \sum_{r_{4+j} \leq P} \sum_{\chi_{4+j} \bmod r_{4+j}}^{*} \max _{|\lambda| \leq 1 / r_{4+j} Q} r_{0}^{-3+\varepsilon}\left|W_{2}\left(\chi_{4+j}, \lambda\right)\right|\left(\max _{|\lambda| \leq 1 / Q}\left|V_{2}(\lambda)\right|\right)^{4-j} \\
& \ll U V^{4} L^{-1},
\end{aligned}
$$

$$
\begin{aligned}
\left|I_{1 j}\right| \ll & L^{c} \sum_{r_{1} \leq P} \sum_{\chi_{1} \bmod r_{1}}^{*} \max _{|\lambda| \leq 1 / Q}\left|W_{1}\left(\chi_{1}, \lambda\right)\right|\left(\max _{|\lambda| \leq 1 / r Q}\left|V_{1}(\lambda)\right|\right) \\
& \times\left(\int_{-1 / Q}^{1 / Q}\left|V_{1}(\lambda)\right|^{2} d \lambda\right) \sum_{r_{5} \leq P} \sum_{\chi_{5} \bmod r_{5}}^{*} \max _{|\lambda| \leq 1 / r_{5} Q}\left|W_{2}\left(\chi_{5}, \lambda\right)\right| \ldots \\
& \ldots \sum_{r_{4+j} \leq P} \sum_{\chi_{4+j} \bmod r_{4+j}}^{*} \max _{|\lambda| \leq 1 / r_{4+j} Q} r_{0}^{-3+\varepsilon}\left|W_{2}\left(\chi_{4+j}, \lambda\right)\right| \\
& \times\left(\max _{|\lambda| \leq 1 / Q}\left|V_{2}(\lambda)\right|\right)^{4-j} \ll U V^{4} L^{-1},
\end{aligned}
$$

$$
\begin{gathered}
\left|I_{0 j}\right| \ll L^{c}\left(\max _{|\lambda| \leq 1 / Q}\left|V_{1}(\lambda)\right|\right)^{2}\left(\int_{-1 / Q}^{1 / Q}\left|V_{1}(\lambda)\right|^{2} d \lambda\right) \\
\times \sum_{r_{5} \leq P} \sum_{\chi_{5} \bmod r_{5}}^{*} \max _{|\lambda| \leq 1 / r_{5} Q}\left|W_{2}\left(\chi_{5}, \lambda\right)\right| \ldots \\
\ldots \sum_{r_{4+j} \leq P} \sum_{\chi_{4+j} \bmod r_{4+j}}^{*} \max _{|\lambda| \leq 1 / r_{4+j} Q} r^{-3+\varepsilon}\left|W_{2}\left(\chi_{4+j}, \lambda\right)\right|\left(\max _{|\lambda| \leq 1 / Q}\left|V_{2}(\lambda)\right|\right)^{4-j} \\
\ll U V^{4} L^{-1}
\end{gathered}
$$

for large $A>0$.

Lemma 2.1 now follows from 4.13, 4.18, 4.19) and 4.23)-4.27).

5. Estimates related to the singular series: the value of $C_{1}$. We need some more notation. Let $C(\chi, a), C(q, a), B\left(n, q ; \chi_{1}, \ldots, \chi_{8}\right)$ and $B(n, q)$ be defined as in (4.1)-4.3). If $\chi_{1}, \ldots, \chi_{8}$ are characters modulo $q$, then we write

$$
A(n, q):=\frac{B(n, q)}{\varphi^{4}(q)}, \quad \mathfrak{S}(n):=\sum_{q=1}^{\infty} A(n, q),
$$


so,

$$
\mathfrak{S}(n):=\sum_{q=1}^{\infty} \frac{1}{\varphi^{8}(q)} \sum_{\substack{a=1 \\(a, q)=1}}^{q}\left(\sum_{\substack{h=1 \\(h, q)=1}}^{q} e\left(\frac{a h^{3}}{q}\right)\right)^{8} e\left(-\frac{a n}{q}\right) .
$$

Proof of Lemma 3.2. It has been shown in [7] that

$$
\mathfrak{S}(n)=\prod_{p}\left(1+\sum_{j=1}^{\gamma} A\left(n, p^{j}\right)\right)
$$

where

$$
p^{\theta} \| k, \quad \gamma= \begin{cases}\theta+2 & \text { if } p=2,2 \mid k, \\ \theta+1 & \text { otherwise. }\end{cases}
$$

When $k=3$, we have

$$
\mathfrak{S}(n)=\{1+A(n, 2)\}\{1+A(n, 3)+A(n, 9)\} \prod_{p \geq 5}\{1+A(n, p)\} .
$$

Let $A(n, q)$ be defined as in (5.1). We will compute $A(n, q)$ for different $q$. For $p=2$, one has

$$
1+A(n, 2)= \begin{cases}2, & n \equiv 0(\bmod 2), \\ 0, & n \neq 0(\bmod 2),\end{cases}
$$

by direct calculation.

For $p=3$,

$$
C(3, a)=\sum_{h=1}^{2} e\left(\frac{a h^{3}}{3}\right)=e\left(\frac{a}{3}\right)+e\left(-\frac{a}{3}\right)=2 \cos \frac{2 \pi a}{3},
$$

so,

$$
\begin{aligned}
A(n, 3) & =\frac{1}{\varphi^{8}(3)} \sum_{a=1}^{2}\left(2 \cos \frac{2 \pi a}{3}\right)^{8} e\left(-\frac{a n}{3}\right) \\
& =\frac{1}{2^{8}}\left(e\left(-\frac{n}{3}\right)+e\left(-\frac{2 n}{3}\right)\right)=\frac{1}{2^{7}} \cos \frac{2 \pi n}{3} .
\end{aligned}
$$

Thus,

$$
\begin{aligned}
& A(n, 3)= \begin{cases}1 / 2^{7}, & n \equiv 0(\bmod 3), \\
-1 / 2^{8}, & n \neq 0(\bmod 3) .\end{cases} \\
& C(9, a)=\sum_{\substack{h=1 \\
(h, 3)=1}}^{9} e\left(\frac{a h^{3}}{9}\right)=3\left(e\left(\frac{a}{9}\right)+e\left(-\frac{a}{9}\right)\right)=6 \cos \frac{2 \pi a}{9}
\end{aligned}
$$


SO,

$$
\begin{aligned}
A(n, 9) & =\frac{1}{\varphi^{8}(9)} \sum_{\substack{a=1 \\
(a, 3)=1}}^{9}\left(6 \cos \frac{2 \pi a}{9}\right)^{8} e\left(-\frac{a n}{9}\right) \\
& =\sum_{\substack{a=1 \\
(a, 3)=1}}^{9}\left(\cos \frac{2 \pi a}{9}\right)^{8} \cos \frac{2 \pi a n}{9} .
\end{aligned}
$$

For different $n, A(n, 9)$ will take five different values, and they satisfy

$$
A(n, 9)>-0.9609375 \text {. }
$$

From (5.4) and 5.5 wet

$$
1+A(n, 3)+A(n, 9)>1-1 / 2^{8}-0.9609375=0.03515625 .
$$

For $p \geq 5$, if $p \equiv 2(\bmod 3)$ and $(p, a)=1$, we have $C(p, a)=-1$, by Lemma 4.3 in Vaughan [29]. So,

$$
\begin{aligned}
B(n, p) & =\sum_{a=1}^{p-1} C^{8}(p, a) e\left(-\frac{a n}{p}\right)=\sum_{a=1}^{p-1} e\left(-\frac{a n}{p}\right) \\
& = \begin{cases}p-1, & p \mid n, \\
-1, & p \nmid n .\end{cases}
\end{aligned}
$$

Thus,

$$
1+A(n, p)=1+\frac{B(n, p)}{\varphi^{8}(p)}= \begin{cases}1+\frac{1}{(p-1)^{7}}, & p \mid n \\ 1-\frac{1}{(p-1)^{8}}, & p \nmid n .\end{cases}
$$

Let $p \equiv 1(\bmod 3)$ with $p \geq 5$. First, when $p=7$,

$$
C(7, a)=\sum_{h=1}^{6} e\left(\frac{a h^{3}}{7}\right)=3\left(e\left(\frac{a}{7}\right)+e\left(-\frac{a}{7}\right)\right)=6 \cos \frac{2 \pi a}{7}
$$

SO,

$$
\begin{aligned}
A(n, 7) & =\frac{1}{\varphi^{8}(7)} \sum_{a=1}^{6}\left(6 \cos \frac{2 \pi a}{7}\right)^{8} e\left(-\frac{a n}{7}\right) \\
& =\sum_{a=1}^{6}\left(\cos \frac{2 \pi a}{7}\right)^{8} \cos \frac{2 \pi a n}{7} .
\end{aligned}
$$

For different $n, A(n, 7)$ will take four different values, and they satisfy

$$
A(n, 7)>-0.75390625 \text {. }
$$


Thus

$$
1+A(n, 7)>1-0.75390625=0.24609375 .
$$

For $p \geq 13$ and $p \equiv 1(\bmod 3)$, noting the elementary estimate (by Lemma 4.3 of [29])

$$
|C(p, a)| \leq 2 \sqrt{p}+1
$$

we get

$$
|B(n, p)|=\left|\sum_{a=1}^{p-1} C^{8}(p, a) e\left(-\frac{a n}{p}\right)\right| \leq(2 \sqrt{p}+1)^{8}(p-1) .
$$

Thus

$$
1+A(n, p)>1-\frac{(2 \sqrt{p}+1)^{8}}{(p-1)^{7}}
$$

Hence

$$
\begin{aligned}
& \prod_{p \geq 5}\{1+A(n, p)\} \geq\{1+A(n, 7)\} \prod_{\substack{p \geq 13 \\
p \equiv 1(\bmod 3)}}\left(1-\frac{(2 \sqrt{p}+1)^{8}}{(p-1)^{7}}\right) \\
& \quad \times \prod_{\substack{p \geq 5, p \equiv 2(\bmod 3) \\
p \mid n}}\left(1+\frac{1}{(p-1)^{7}}\right) \prod_{\substack{p \geq 5, p \equiv 2(\bmod 3) \\
p \nmid n}}\left(1-\frac{1}{(p-1)^{8}}\right) \\
& \geq\{1+A(n, 7)\} \\
& \quad \prod_{p \geq 13}\left(1-\frac{(2 \sqrt{p}+1)^{8}}{(p-1)^{7}}\right) \\
& \quad \prod_{p \geq 5, p \equiv 2(\bmod 3)}\left(1-\frac{1}{(p-1)^{2}}\right) .
\end{aligned}
$$

To estimate the products above, we apply the elementary inequality

$$
\frac{(2 \sqrt{p}+1)^{8}}{(p-1)^{7}}<\frac{1}{(p-1)^{2}} \quad \text { for } p \geq 324 .
$$

Thus we have

$$
\begin{gathered}
\prod_{p \geq 5}\{1+A(n, p)\} \geq\{1+A(n, 7)\} \prod_{\substack{13 \leq p \leq 323 \\
p \equiv 1(\bmod 3)}}\left(1-\frac{(2 \sqrt{p}+1)^{8}}{(p-1)^{7}}\right) \\
\times \prod_{\substack{p \geq 324 \\
p \equiv 1(\bmod 3)}}\left(1-\frac{1}{(p-1)^{2}}\right) \prod_{\substack{p \geq 5 \\
p \equiv 2(\bmod 3)}}\left(1-\frac{1}{(p-1)^{2}}\right)
\end{gathered}
$$




$$
\begin{aligned}
&=\{1+A(n, 7)\} \prod_{\substack{13 \leq p \leq 323 \\
p \equiv 1(\bmod 3)}}\left(1-\frac{(2 \sqrt{p}+1)^{8}}{(p-1)^{7}}\right) \\
& \times \prod_{p=3,7}\left(1-\frac{1}{(p-1)^{2}}\right)^{-1} \prod_{\substack{13 \leq p \leq 323 \\
p \equiv 1(\bmod 3)}}\left(1-\frac{1}{(p-1)^{2}}\right)^{-1} \\
& \times \prod_{p \geq 3}\left(1-\frac{1}{(p-1)^{2}}\right) \\
& \geq 0.24609375 \cdot \frac{4}{3} \cdot \frac{36}{35} \cdot 0.35608989538 \cdot 0.6601 \\
& \geq 0.079331042229,
\end{aligned}
$$

where we have used $\prod_{p \geq 3}\left(1-(p-1)^{-2}\right)=0.6601 \ldots$ (see [5]).

This in combination with (5.2), (5.3), 5.6), (5.12) ensures that

$$
\mathfrak{S}(n)>0.00557795824
$$

when $n \equiv 0(\bmod 2)$. The proof is complete.

6. Upper bounds of $J_{i}(g)$ and $K(g)$ : proof of Lemmas 4.2-4.4. Lemmas 4.2 and 4.3 are similar to those in Section 5 in Liu and Liu [19], and the choices of $P, Q$ defined in (2.1) are acceptable in these lemmas. A similar proof can also be found in [24], so we omit the details. Here we only give the proof of Lemma 4.4 .

In the proof, we need a mean value theorem of Choi and Kumchev [2]:

Lemma 6.1. Let $l$ be a positive integer, $R, T, X \geq 1$ and $\kappa=1 / \log X$. Then there is an absolute positive constant $c$ such that

$$
\sum_{r \sim R} \sum_{\chi \mid r}^{*} \int_{\chi \bmod r}^{T}\left|\sum_{X<n \leq 2 X} \frac{\Lambda(n) \chi(n)}{n^{\kappa+i \tau}}\right| d \tau \ll\left(l^{-1} R^{2} T X^{11 / 20}+X\right)(\log R T X)^{c}
$$

where the implied constant is absolute.

In order to use Lemma 6.1 effectively, we need a lemma of [15]:

Lemma 6.2. Let $\chi$ be a Dirichlet character modulo $r$. Let $2 \leq X<Y$ $\leq 2 X, T_{0}=(\log (Y / X))^{-1}, T=X^{4}$ and $\kappa=1 / \log X$. Define

$$
F(s, \chi)=\sum_{X \leq n \leq 2 X} \Lambda(n) \chi(n) n^{-s} .
$$


Then

$$
\begin{aligned}
\sum_{X \leq n \leq 2 X} \Lambda(n) \chi(n) \ll & \log (Y / X) \int_{|\tau| \leq T_{0}}|F(\kappa+i \tau, \chi)| d \tau \\
& +\int_{T_{0}<|\tau| \leq T} \frac{|F(\kappa+i \tau, \chi)|}{|\tau|} d \tau+1 .
\end{aligned}
$$

The implied constants are absolute.

Proof of Lemma 4.4. Introduce

$$
\widehat{W}_{1}(\chi, \lambda):=\sum_{m \sim U} \Lambda(m) \chi(m) e\left(\lambda m^{3}\right)-\delta_{\chi} \sum_{m \sim U} e\left(\lambda m^{3}\right) .
$$

When we replace $W_{1}(\chi, \lambda)$ by $\widehat{W}_{1}(\chi, \lambda)$, the error is

$$
\widehat{W}_{1}(\chi, \lambda)-W_{1}(\chi, \lambda) \ll U^{1 / 2} \text {. }
$$

Thus the resulting error of $K(g)$ is

$$
\begin{aligned}
& \ll[g, r]^{-3+\varepsilon} \frac{r^{1 / 2} U^{1 / 2}}{Q^{1 / 2}} \ll g^{-3+\varepsilon} \frac{U^{1 / 2}}{Q^{1 / 2}} \sum_{\substack{l \leq P \\
l \mid g}} l^{3-\varepsilon} \sum_{\substack{r \leq P \\
l \mid r}} r^{-5 / 2+\varepsilon} \\
& \ll g^{-3+\varepsilon} U^{-1 / 2} L^{c} .
\end{aligned}
$$

Here, in the last step, we need the definition of $P$ and $Q$ in 2.1.

Thus to establish Lemma 4.4, it suffices to show that

$$
\sum_{r \sim R}[g, r]^{-3+\varepsilon} \sum_{\chi \bmod r}^{*}\left(\int_{-1 / r Q}^{1 / r Q}\left|\widehat{W}_{1}(\chi, \lambda)\right|^{2} d \lambda\right)^{1 / 2} \ll g^{-3+\varepsilon} U^{-1 / 2} L^{c}
$$

for any $R \leq P$ and some $c>0$.

By Gallagher's lemma ([4, Lemma 1]), we have

$$
\begin{aligned}
\int_{-1 / r Q}^{1 / r Q}\left|\widehat{W}_{1}(\chi, \lambda)\right|^{2} d \lambda & \\
& \ll\left(\frac{1}{R Q}\right)^{2} \int_{-\infty}^{\infty}\left|\sum_{\substack{v \leq m^{3} \leq v+r Q \\
m \sim U}}\left(\Lambda(m) \chi(m)-\delta_{\chi}\right)\right|^{2} d v \\
& \ll\left(\frac{1}{R Q}\right)^{2} \int_{U^{3}-r Q}^{(2 U)^{3}}\left|\sum_{X<m \leq Y}^{\substack{m \sim m \\
X<}}\left(\Lambda(m) \chi(m)-\delta_{\chi}\right)\right|^{2} d v,
\end{aligned}
$$

where

$$
X:=\max \left\{v^{1 / 3}, U\right\}, \quad Y:=\min \left\{(v+r Q)^{1 / 3}, 2 U\right\} .
$$


If $R=1$, we have

$$
\begin{aligned}
\sum_{X<n \leq Y}\left(\Lambda(m) \chi(m)-\delta_{\chi}\right) & =\sum_{X<m \leq Y}(\Lambda(m)-1) \\
& \ll(Y-X) L \ll U^{-2} Q L .
\end{aligned}
$$

This contributes to 6.3 the quantity

$$
g^{-3+\varepsilon}\left(\frac{1}{Q^{2}} \cdot U^{3} \cdot U^{-4} Q^{2}\right)^{1 / 2} L \ll g^{-3+\varepsilon} U^{-1 / 2} L,
$$

which is acceptable.

For $R \geq 2$ and $r \sim R$, we have $\delta_{\chi}=0$. Thus, we can apply (6.1) to obtain

$$
\begin{aligned}
\int_{-1 / r Q}^{1 / r Q}\left|\widehat{W}_{1}(\chi, \lambda)\right|^{2} d \lambda \ll \frac{1}{U^{3}}\left(\int_{|\tau| \leq T_{0}}|F(\kappa+i \tau, \chi)| d \tau\right)^{2} \\
+\frac{U^{3}}{(R Q)^{2}}\left(\int_{T_{0}<|\tau| \leq T} \frac{|F(\kappa+i \tau, \chi)|}{|\tau|} d \tau\right)^{2}+\frac{U^{3}}{(R Q)^{2}},
\end{aligned}
$$

since $T_{0}^{-1}=\log (Y / X) \asymp R Q / U^{3}$.

Therefore, the contribution of the first term of (6.7) to the left-hand side of 6.3 is

$$
\begin{aligned}
& \ll g^{-3+\varepsilon} U^{-3 / 2} \sum_{l \leq 2 R}\left(\frac{R}{l}\right)^{-3+\varepsilon}\left(l^{-1} R^{2} T_{0} U^{11 / 20}+U\right) L^{c} \\
& \ll g^{-3+\varepsilon} U^{-3 / 2}\left(R^{-1+\varepsilon} \sum_{l \leq 2 R} l^{2-\varepsilon} T_{0} U^{11 / 20}+U\right) L^{c} \\
& \ll g^{-3+\varepsilon} U^{-1 / 2} L^{c}, \quad{ }_{l \mid g}
\end{aligned}
$$

which is acceptable by the definition of $Q$.

Set

$$
M\left(l, R, T^{\prime}, U\right):=\sum_{r \sim R} \sum_{\chi \mid r}^{*} \int_{T^{\prime}}^{2 T^{\prime}}|F(\kappa+i \tau, \chi)| d \tau .
$$

The contribution of the second term of 6.7$)$ to the left-hand side of $(6.3)$ is

$$
\begin{aligned}
& \ll g^{-3+\varepsilon} U^{3 / 2}(R Q)^{-1} \sum_{l \leq 2 R}\left(\frac{R}{l}\right)^{-3+\varepsilon} \max _{T_{0} \leq T^{\prime} \leq T} T^{-1} M\left(l, R, T^{\prime}, U\right) \\
& \ll g^{-3+\varepsilon} U^{3 / 2}(R Q)^{-1} \sum_{l \leq 2 R}\left(\frac{R}{l}\right)^{-3+\varepsilon}\left(l^{-1} R^{2} U^{11 / 20}+T_{0}^{-1} U\right) L^{c} \\
& \ll g^{-3+\varepsilon} U^{-1 / 2} L^{c}, \quad \quad l \mid g
\end{aligned}
$$

which is acceptable by the definition of $Q$. 
Finally, the contribution of the last term of (6.7) to the left-hand side of 6.3 is

$$
\ll g^{-3+\varepsilon} U^{3 / 2}(R Q)^{-1} \sum_{\substack{l \leq 2 R \\ l \mid g}}\left(\frac{R}{l}\right)^{-3+\varepsilon} \ll g^{-3+\varepsilon} U^{-1 / 2} L^{c} .
$$

Now Lemma 4.4 follows from 6.2, 6.3, 6.6 and 6.8 6.10 .

Acknowledgments. This work is partly supported by the key project of the National Natural Science Foundation of China (Grant No. 11031004), Shandong Province Natural Science Foundation (Grant No. ZR2009AM007), and IIFSDU. The authors would like to express their thanks to the referee for many useful suggestions and comments on the manuscript.

\section{References}

[1] J. Brüdern, A sieve approach to the Waring-Goldbach problem (I): sums of four cubes, Ann. Sci. École Norm. Sup. 28 (1995), 461-476 .

[2] S. K. K. Choi and A.V. Kumchev, Mean values of Dirichlet polynomials and applications to linear equations with prime variables, Acta Arith. 123 (2006), 125-142.

[3] P. X. Gallagher, Primes and powers of 2, Invent. Math. 29 (1975), 125-142.

[4] - A large sieve density estimate near $\sigma=1$, ibid. 11 (1970), 329-339.

[5] H. Halberstam and H. E. Richert, Sieve Methods, Academic Press, London, 1974.

[6] D. R. Heath-Brown and J.-C. Puchta, Integers represented as a sum of primes and powers of two, Asian J. Math. 6 (2002), 535-565.

[7] L. K. Hua, Some results in the additive prime number theory, Quart. J. Math. (Oxford) 9 (1938), 68-80.

[8] -, Additive Theory of Prime Numbers, Science Press, Beijing, 1957 (in Chinese); English transl.: Amer. Math. Soc., Providence, RI, 1965.

[9] K. Kawada, Note on the sum of cubes of primes and an almost prime, Arch. Math. (Basel) 69 (1997), 13-19.

[10] A. V. Kumchev, On the Waring-Goldbach problem: exceptional sets for sums of cubes and higher powers, Canad. J. Math. 57 (2005), 298-327.

[11] M. C. Leung and M. C. Liu, On generalized quadratic equations in three prime variables, Monatsh. Math. 115 (1993), 133-169.

[12] H. Z. Li, The number of powers of 2 in a representation of large even integers by sums of such powers and of two primes, Acta Arith. 92 (2000), 229-237.

[13] - , The number of powers of 2 in a representation of large even integers by sums of such powers and of two primes (II), ibid. 96 (2001), 369-379.

[14] - Four prime squares and powers of 2, ibid. 125 (2006), 383-391.

[15] H. Z. Li and J. Wu, Sums of almost equal cubes, Funct. Approx. Comment. Math. 38 (2008), 49-65.

[16] Yu. V. Linnik, Prime numbers and powers of two, Trudy Mat. Inst. Steklov. 38 (1951), 151-169 (in Russian).

[17] - Addition of prime numbers with powers of one and the same number, Mat. Sb. (N.S.) 32 (1953), 3-60 (in Russian). 
[18] J. Y. Liu and M. C. Liu, Representation of even integers as sums of squares of primes and powers of 2, J. Number Theory 83 (2000), 202-225.

[19] —, - , The exceptional set in four prime squares problem, Illinois J. Math. 44 (2000), $272-293$.

[20] - - - Representation of even integers by cubes of primes and powers of 2, Acta Math. Hungar. 91 (3) (2001), 217-243.

[21] J. Y. Liu, M. C. Liu and T. Z. Wang, The number of powers of 2 in a representation of large even integers (II), Sci. China Ser. A 41 (1998), 1255-1271.

[22] J. Y. Liu, M. C. Liu and T. Zhan, Squares of primes and powers of 2, Monatsh. Math. 128 (1999), 283-313.

[23] J. Y. Liu and G. S. Lü, Four squares of primes and 165 powers of 2, Acta Arith. 114 (2004), 55-70.

[24] G. S. Lü and Y. F. Xu, Hua's theorem with nine almost equal prime variables, Acta Math. Hungar. 116 (2007), 309-326.

[25] J. Pintz and I. Z. Ruzsa, On Linnik's approximation to Goldbach's problem, I, Acta Arith. 109 (2003), 169-194.

[26] X. M. Ren, Density of integers that are the sum of four cubes of primes, Chin. Ann. Math. (Ser. B) 22 (2001), 233-242.

[27] —, Sums of four cubes of primes, J. Number Theory 98 (2003), 156-171.

[28] K. F. Roth, On Waring's problem for cubes, Proc. London Math. Soc. 53 (1951), 268-279.

[29] R. C. Vaughan, The Hardy-Littlewood Method, 2nd ed., Cambridge Univ. Press, 1997.

[30] T. Z. Wang, On Linnik's almost Goldbach theorem, Sci. China Ser. A 42 (1999), $1155-1172$.

[31] T. D. Wooley, Slim exceptional sets for sums of cubes, Canad. J. Math. 54 (2002), 417-448.

Zhixin Liu, Guangshi Lü

School of Mathematics

Shandong University

Ji'nan, 250100, P.R. China

E-mail: zxliu@mail.sdu.edu.cn

gslv@sdu.edu.cn

Received on 8.11.2009

and in revised form on 14.4.2010 